\title{
Accuracy of genomic predictions of residual feed intake and 250-day body weight in growing heifers using 625,000 single nucleotide polymorphism markers
} \author{
Y. J. Williams,, R. J. Spelman, $\neq$ and B. J. Hayes ${ }^{\star} \dagger$ \\ *Biosciences Research Division, Department of Primary Industries Victoria, Bundoora, VIC 3083, Australia \\ †Dairy Futures Cooperative Research Centre, Bundoora, VIC 3083, Australia \\ †LIC, Private Bag 3016, Hamilton 3240, New Zealand \\ $\S$ ViaLactia Biosciences (NZ) Ltd., PO Box 109-185, Auckland, New Zealand \\ \#DairyNZ, Private Bag 3221, Hamilton 3240, New Zealand \\ IIDepartment of Primary Industries, Ellinbank, VIC 3820, Australia \\ IDepartment of Primary Industries, Tatura, VIC 3616, Australia
}

J. E. Pryce, ${ }^{*} \dagger^{1}$ J. Arias,‡ P. J. Bowman, ${ }^{\dagger}$ S. R. Davis,§ K. A. Macdonald,\# G. C. Waghorn,\# W. J. Wales,॥

\begin{abstract}
Feed makes up a large proportion of variable costs in dairying. For this reason, selection for traits associated with feed conversion efficiency should lead to greater profitability of dairying. Residual feed intake (RFI) is the difference between actual and predicted feed intakes and is a useful selection criterion for greater feed efficiency. However, measuring individual feed intakes on a large scale is prohibitively expensive. A panel of DNA markers explaining genetic variation in this trait would enable cost-effective genomic selection for this trait. With the aim of enabling genomic selection for RFI, we used data from almost 2,000 heifers measured for growth rate and feed intake in Australia (AU) and New Zealand (NZ) genotyped for 625,000 single nucleotide polymorphism (SNP) markers. Substantial variation in RFI and 250-d body weight (BW250) was demonstrated. Heritabilities of RFI and BW250 estimated using genomic relationships among the heifers were 0.22 and 0.28 in $\mathrm{AU}$ heifers and 0.38 and 0.44 in NZ heifers, respectively. Genomic breeding values for RFI and BW250 were derived using genomic BLUP and 2 Bayesian methods (BayesA, BayesMulti). The accuracies of genomic breeding values for RFI were evaluated using cross-validation. When 624,930 SNP were used to derive the prediction equation, the accuracies averaged 0.37 and 0.31 for RFI in $\mathrm{AU}$ and $\mathrm{NZ}$ validation data sets, respectively, and 0.40 and 0.25 for BW250 in $\mathrm{AU}$ and NZ, respectively. The greatest advantage of using the full 624,930 SNP over a reduced panel of 36,673 SNP (the widely used BovineSNP50 array) was when the reference population included only animals
\end{abstract}

Received June 16, 2011

Accepted November 18, 2011.

${ }^{1}$ Corresponding author: jennie.pryce@dpi.vic.gov.au from either the AU or the NZ experiment. Finally, the Bayesian methods were also used for quantitative trait loci detection. On chromosome 14 at around $25 \mathrm{Mb}$, several SNP closest to PLAG1 (a gene believed to affect stature in humans and cattle) had an effect on BW250 in both $\mathrm{AU}$ and $\mathrm{NZ}$ populations. In addition, $8 \mathrm{SNP}$ with large effects on RFI were located on chromosome 14 at around $35.7 \mathrm{Mb}$. These SNP may be associated with the gene NCOA2, which has a role in controlling energy metabolism.

Key words: feed conversion efficiency, residual feed intake, genomic selection

\section{INTRODUCTION}

Feed makes up a large percentage of variable costs in dairy farming, with estimates of between 43 and $67 \%$ of total farm costs in Australia (Ho et al., 2005) and similar percentages in other countries (e.g., Simm et al., 1994). Consequently, genetic improvement of traits associated with feed conversion efficiency could be of considerable economic importance. Other potential benefits of more efficient cattle have been identified recently, such as an association with lower methane emissions (Hegarty et al., 2007).

Selection for residual feed intake (RFI) is one option to improve feed efficiency. In growing cattle, RFI is usually defined as the difference between an animal's actual feed intake and its expected feed intake based on its size and growth over a specific period. Significant genetic variation in RFI of up to $30 \%$ has been demonstrated in growing beef cattle (Arthur et al., 2004; Nkrumah et al., 2007a). In dairy cattle, however, measurements of RFI are complicated by dynamic changes in BW and body condition during lactation. Evidence already exists to show that selection for RFI in growing animals is correlated to RFI in mature breeding and 
lactating animals (Nieuwhof et al., 1992). Therefore, measuring RFI in growing heifers rather than lactating cows is attractive, as the problems associated with negative energy balance due to mobilization of body tissue generally do not exist in nonlactating dairy heifers (Williams et al., 2011).

However, even measuring RFI in growing heifers is still too expensive as an industry-wide strategy to improve feed conversion efficiency of dairy cattle. Genomic selection is an appealing alternative. Genomic selection involves assembling a reference population of individuals with phenotypic measurements for the target trait (in this case RFI), genotyping these individuals for a panel of genome-wide SNP, and then using this information to derive a prediction equation of the effects of the markers on the trait. The equation can then be used to predict breeding values for RFI in any genotyped animal that is a candidate for selection (Meuwissen et al., 2001).

The aim of this study was to calculate the accuracy of genomic estimated breeding values (GEBV) for RFI and BW at $250 \mathrm{~d}$ (BW250; selected to be a comparison trait), using a reference population of heifers from Australia (AU) and New Zealand (NZ). Three methods were used to predict genomic breeding values: genomic BLUP (GBLUP), which assumes that all SNP have a small effect and these effects are normally distributed, and 2 Bayesian methods, one with a prior assumption that the SNP effects follow a $t$-distribution, and a second method in which the effect of some SNP could be zero. An additional aim was to use Bayesian genomic selection as a way of locating QTL in a genome-wide association study (GWAS), where all SNP were fitted simultaneously.

\section{MATERIALS AND METHODS}

\section{Animals and Facilities}

With a collaborative effort between research organizations in $\mathrm{AU}$ and $\mathrm{NZ}$, resources were available to take measurements required to calculate RFI on 2,000 Holstein heifer calves, approximately 1,000 in each country. The AU trial was carried out over 2 yr (2009 and 2010) in Rutherglen, Victoria $\left(36^{\circ} 06^{\prime} \mathrm{S}, 146^{\circ} 30^{\prime} \mathrm{E}\right)$ and included 2 spring-born and 1 autumn-born cohort of calves (see Williams et al., 2011 for a full description). The NZ trial was carried out at Hawera, Taranaki $\left(39^{\circ} 36^{\prime} 41 \mathrm{~S}, 174^{\circ} 18^{\prime} 28 \mathrm{E}\right)$ over 3 yr (2008 to 2010 ), comprised entirely spring-born calves in 3 cohorts or 5 groups (in the last $2 \mathrm{yr}$, the cohort was divided into 2 groups for management reasons and the groups were tested sequentially). In both countries, calves were tested when they were approximately 6 to 8 mo old.

The feed offered to the calves was alfalfa (Medicago sativa) cubes available ad libitum. Dried alfalfa was chosen because it is similar to pasture (the predominant feed in both AU and NZ) but has consistent nutritive values (Williams et al., 2011). Both the AU and NZ trials used electronic feed intake measuring devices made by Gallagher Animal Management Systems (Hamilton, New Zealand). The feed intake units were hard-wired to data loggers, so data were relayed $24 \mathrm{~h} / \mathrm{d}$ for the duration of the trial. Full details of the phenotype data collection and data editing techniques can be found in Williams et al. (2011) for AU data and in Carnie et al. (2010) and Waghorn et al. (2012) for NZ data.

\section{Phenotypes}

In calves of the age used in this trial, growth rates (average daily gain in $\mathrm{BW}$ ) were observed to be very close to linear (Williams et al., 2011). Therefore, to calculate growth rates, or the average daily gain $(B W \Delta)$ and mid-point BW $(m B W)$ for the 6- to 7-wk measurement period, a linear regression of body weight $(B W$; $\mathrm{kg})$ on day of test $(D O T)$ was calculated for each animal: $B W=\mu+b D O T+e$, where $\mu$ is the overall mean effect, $b$ is the regression coefficient (i.e., the growth rate; $\mathrm{kg} / \mathrm{d} ; B W \Delta)$, and $e$ is the residual error term. Then, BW250 was calculated by solving the estimated regression equation for the weight on test corresponding to $250 \mathrm{~d}$ of age for each heifer in turn.

Dry matter intake was calculated daily throughout the trial period (Williams et al., 2011). Similar methods of editing and data quality methods were applied to DMI and BW data in both countries. A more detailed description is given by Williams et al. (2011).

Calculation of RFI in Australia. Residual feed intake was calculated for each cohort of heifers separately. In this study, prediction of mean DMI ( $m D M I)$ was done using mid-point $\mathrm{BW}(m B W)$ and growth rate $(B W \Delta)$. Variation in age was adjusted for using a quadratic regression on age at the start of the trial. The AU heifers were born over a period of around 12 wk within each cohort. The NZ heifers were purchased at birth and reared in the same environment per cohort, whereas the AU heifers were on loan from dairy farms. Therefore, for AU heifers, the effect of farm was adjusted for to account for the variation observed in the farm effect for DMI and growth rate. The effect of pen was not significant and so was not included, probably because in most cases it was confounded with 
farm; as far as possible, calves were kept with their farm contemporaries:

$$
\begin{aligned}
m D M I & =\mu+b_{1} B W \Delta+b_{2} m B W+\text { farm } \\
& +b_{3} a g e+b_{4} a g e^{2}+R F I,
\end{aligned}
$$

where $\mu=$ the overall mean effect and $b_{1}, b_{2}, b_{3}$, and $b_{4}$ are partial regression coefficients. Note: RFI is the residual error term in this equation.

Calculation of RFI in New Zealand. Residual feed intake was estimated as the residual after multiple regression of intake on $B W \Delta(\mathrm{kg} / \mathrm{d})$ and mean $\mathrm{BW}$ at the trial mid-point. The heifers used in the NZ trial cohorts were born over a short interval of around 3 to $7 \mathrm{wk}$; therefore, age was not statistically significant in the model fitted and was omitted from the final model:

$$
m D M I=\mu+b_{1} B W \Delta+b_{2} m B W+R F I .
$$

\section{Genotype Data Quality Control}

Nine hundred three AU heifers and 1,034 NZ heifers were genotyped (1,937 total) with the Illumina High Density Bovine SNP chip (Illumina Inc., San Diego, CA; www.illumina.com/agriculture), which comprises 777,963 SNP markers. The SNP were mapped to the UMD 3.1 build assembled by the Center for Bioinformatics and Computational Biology at the University of Maryland (http://www.cbcb.umd.edu/research/ bos_taurus_assembly.shtml). Stringent quality control procedures were applied to the data. These included the use of the Illumina Genetrain (GC) score, which describes the performance of genotyping each SNP in each individual. Genotype calls with GC $>0.6$ are high quality; below this value they were excluded. In the combined (AU and NZ) data set, 650,934 SNP had $>90 \%$ of animals genotyped at GC $>0.6$, with 343 mitochondrial SNP, 1,124 Y SNP, and 1,735 unmapped SNP excluded. Some 55 SNP with duplicate map positions were removed and thus 650,880 SNP remained. Forty-eight individuals with fewer than $90 \%$ of SNP genotyped at GC $<0.6$ were removed. Across the remaining samples, $99.6 \%$ of SNP were genotyped at GC $>0.6$. Animals with excess heterozygosity $(>0.4)$ were removed, as this is a good indicator of sample contamination. Five animals were identified with excess heterozygosity; however, all had already been removed in the previous step (i.e., $>90 \%$ of SNP genotyped). The final stage of filtering was for SNP with very low minor allele frequency; 16,316 SNP had a minor allele frequency $<0.5 \%$ and were removed. Single nucleotide polymorphisms that were very extreme with respect to their
Hardy-Weinberg equilibrium can indicate genotyping errors; the 9,632 SNP that had probabilities calculated from $\chi^{2}$ tests of $<0.01$ were removed (these were, in almost all cases, SNP with both homozygous genotypes observed but no heterozygotes). In total, 624,930 SNP passed all criteria. The error rate was checked using 4 duplicate samples and was very low (between 0.007 and $0.06 \%$ ). Finally, all individual genotype calls with GC $<0.6$ were set to missing. All missing genotypes were imputed by using the BEAGLE program (Browning and Browning, 2009).

Two data sets of SNP were constructed. One consisted of all $624,930 \mathrm{SNP}$ and referred to as the $600 \mathrm{k}$ SNP panel. The other set, referred to as the 40k SNP panel, consisted of $36,673 \mathrm{SNP}$ and was a subset of the 624,330 SNP that were present on the BovineSNP50 BeadChip (Illumina; Matukumalli et al., 2009). In both data sets, 1,782 animals passed both the phenotype and genotype editing criteria.

\section{Genomic Predictions}

$\boldsymbol{G B L} \boldsymbol{U P}$. If many QTL exist whose effects are assumed to be normally distributed with constant variance, then the expected pedigree relationship matrix can be replaced with the realized or genomic relationship matrix $(\mathbf{G})$ estimated from DNA markers in the mixed model equations used to calculate breeding values (e.g., VanRaden, 2008; Yang et al., 2010). One model was used for all traits:

$$
\begin{aligned}
y_{\text {hijkl }} & =\mu+\text { country }_{h}+\text { group }_{i}+\text { farm }_{j} \\
& + \text { b }_{1} \text { age }_{\text {hijkl }}+b_{2} \text { NAhol }_{h i j k l}+g_{k}+e_{\text {hijkl }},
\end{aligned}
$$

where $\mu$ was the overall mean; country $y_{h}$ was either AU or NZ; group $_{i}$ was for NZ data only and included 5 levels (1 cohort in 2008, 2 cohorts each in 2009 and 2010); farm $_{j}$ was the $j$ th farm of origin (nested within year) for AU data only; $b_{1}$ was the regression on the covariate age, which was the age on starting the test period deviated from the mean age of heifers on trial at the same time; $b_{2}$ was the regression coefficient on North American Holstein percentage ( $\mathrm{NAHol \%}$, fitted to NZ data only); and finally $g_{k}$ was the genomic breeding value of the $k$ th heifer. ASReml was used for the GBLUP analyses (Gilmour et al., 2006).

In addition, the heritabilities of RFI and BW250 were estimated using bivariate models with the same effects as fitted in the previous univariate model using ASReml (Gilmour et al., 2006). In this analysis, RFI and BW250 were considered different traits in AU and NZ so that the genetic correlation of the same trait measured in AU and NZ could be estimated. 
BayesA. This approach for calculating genomic breeding values assumes that individual SNP effects follow a Student's t-distribution (Meuwissen et al., 2001). Using a $t$-distribution of SNP effects rather than a normal distribution allows for a higher probability of moderate to large SNP effects than GBLUP. To implement BayesA, phenotypes were first precorrected for all the fixed effects. The model used to precorrect the phenotypes was almost identical to the GBLUP model and correspondingly included the following fixed effects: country, age, group, farm, and North American Holstein percentage. The model used for BayesA was

$$
\mathbf{y}=\mathbf{1}_{\mathbf{n}}{ }^{\prime} \mu+\mathbf{X} \mathbf{u}+\mathbf{Z} \mathbf{v}+\mathbf{e},
$$

where $\mathbf{y}$ is a vector of phenotypes from $n$ individuals corrected for fixed effects; $\mathbf{1}_{\mathbf{n}}$ is a vector of 1 s of length $n ; \mathbf{X}$ is an $(n \times m)$ design matrix allocating records to $m$ marker effects with element $X_{i j}=0,1$, or 2 if the genotype of animal $i$ at SNP $j$ is 11,12 , or 22 , respectively; $\mathbf{u}$ is an $(m \times 1)$ vector of SNP effects; $\mathbf{e}$ is a vector of random deviates, where $\sigma_{e}^{2}$ is the error variance, $v_{i}$ is the polygenic breeding value of the $i$ th animal, with variance $\mathbf{A} \sigma_{a}^{2}$, where $\mathbf{A}$ is the average relationship matrix. In this case, only sire was fitted. The prior for $\sigma_{u i}^{2}$ was an inverse chi-squared distribution with 4.012 degrees of freedom. Gibbs sampling was used to derive estimates of the SNP and other effects.

BayesMulti. Another possible assumption about SNP effects is that many have no effect, as they are not in linkage disequilibrium (LD) with any of the mutations that explain the variation in RFI or BW250. The BayesMulti method is an extension of Bayesian SSVS (Verbyla et al., 2009), except that SNP effects are assumed to come from a series of normal distributions (hence, BayesMulti for Bayes Multinomial). Fifty-five percent of the SNP were assumed to have no effect, $40 \%$ were assumed to have a very small effect (explaining $0.003 \%$ of the variance per SNP), $4.9 \%$ were assumed to have a small effect (explaining $0.03 \%$ of the variance), and $0.1 \%$ were assumed to have a small to moderate effect ( $3 \%$ of the variance). Investigations using different values for prior proportions and variances showed that accuracies of genomic prediction were insensitive to the prior values over a wide range of values. For the BayesMulti analyses, the same model was fitted as used for BayesA.

Calculating the Accuracy of Genomic Breeding Values. The main aim of this analysis was to determine how well the genomic breeding values predict the true breeding values of individual animals. If the true breeding values of individuals were known, the accuracy of the genomic breeding values would be the correlation between the genomic breeding values and the true breeding values. In practice, the true breeding values are unknown, and the only data available are phenotypes, which are made up of the effect of the true breeding value and the environmental effect. Given this, the accuracy of the genomic breeding values was derived as follows.

From the 6 cohorts used in the trial, a single cohort (the validation cohort; AU1, AU2, or AU3 for the $3 \mathrm{AU}$ trials; NZ1, NZ2, or NZ3 for the 3 New Zealand trials) was removed from the data set. The SNP effects for either RFI or BW250 were calculated in the reduced data set using the methods above. Using the estimated SNP effects, a vector of genomic estimated breeding values (GEBV) was calculated for the heifers in the validation cohort, $\mathbf{G E B V}=\mathbf{X} \hat{\mathbf{u}}$, where $\mathbf{X}$ is a matrix of the heifers' genotypes, and $\hat{\mathbf{u}}$ is the vector of SNP effects predicted by either BayesA or BayesMulti. For GBLUP, GEBV were predicted using the system of equations described earlier. For each cohort removed from the data set, the GEBV was then correlated with a vector of phenotypes (Phenotype) of RFI or BW250 heifers in that cohort, corrected for the fixed effects described above. This gave r(GEBV, Phenotype). However, the accuracy of GEBV should be the correlation between GEBV and a vector of true breeding value (TBV). The accuracy of TBV was approximated as $\mathrm{r}$ (GEBV,Phenotype)/h, where $\mathrm{h}$ was the square root of the heritability of either RFI or BWT250. A mean accuracy was also calculated for each country, by averaging across the cohorts.

\section{GWAS}

Fitting all SNP together in Bayesian models can locate putative QTL in a GWAS. Fitting the SNP simultaneously, rather than individually, is potentially advantageous, because it should improve the precision of mapping (Pryce et al., 2010). The BayesA and BayesMulti SNP effects estimated using AU and NZ data sets separately and together were used to locate putative QTL.

Generally, in GWAS, when SNP are fitted individually, $P$-values are used to determine whether a SNP has an association with a given phenotype. However, as $P$-values are not a product of Bayesian methods, other methods of validating SNP are required. The 1,000 largest SNP effects, ranked on absolute value were selected (i.e., SNP that had effects that were among the largest $0.0016 \%$ out of all SNP in the analysis).

\section{RESULTS}

\section{Heritability}

The heritabilities estimated using the genomic relationship matrix (Table 1) were $0.22(0.07)$ and 0.38 
Table 1. Phenotypic standard deviations (SD) and heritabilities $\left(\mathrm{h}^{2}\right)$ estimated with the genomic relationship matrix for residual feed intake (RFI) and 250-d BW (BW250) estimated in data from Australia and New Zealand

\begin{tabular}{llccc}
\hline Country & Trait & Mean $(\mathrm{kg})$ & $\mathrm{SD}(\mathrm{kg})$ & $\mathrm{h}^{2}(\mathrm{SE})$ \\
\hline Australia & RFI & 0 & 0.42 & $0.22(0.07)$ \\
New Zealand & RFI & 0 & 0.50 & $0.38(0.09)$ \\
Australia & BW250 & 241.5 & 42.0 & $0.28(0.07)$ \\
New Zealand & BW250 & 189.7 & 17.9 & $0.44(0.10)$ \\
\hline
\end{tabular}

(0.09) for RFI estimated in AU and NZ heifers, respectively. For BW250, the heritabilities were $0.28(0.07)$ and 0.44 (0.09) estimated in AU and NZ heifers, respectively. The genetic correlations estimated between countries had large associated standard errors for both traits. The Akaike information criteria (AIC) of the models where the genetic correlation between countries could vary were compared with models where the genetic correlations were fixed to 0 and 1 . The lowest $\mathrm{AIC}$, and therefore the preferred model, was when the correlation was fixed to 1 for both RFI and BW250.

\section{Accuracy of Genomic Prediction}

The accuracy of GEBV for RFI was 0.37 in the AU cohorts when averaged across the 3 cohorts and models using the 600k SNP panel (Table 2). The accuracy of GEBV in the NZ data was lower (0.31). The accuracy of predicting RFI with the 40k SNP panel was slightly lower in the AU data when the $600 \mathrm{k}$ SNP panel was used (0.36). In NZ, the accuracy of the GEBV was also lower $(0.29)$.

An advantage was found in using Bayesian methods (BayesA and BayesMulti) over GBLUP for RFI in AU heifers. This was not observed for RFI or BW250 in NZ heifers (a small advantage was observed for BW250 in $\mathrm{AU})$.

The average accuracy of predicting BW250 in the 3 cohorts with the 600k SNP panel was 0.40 (AU) and
0.25 (New Zealand; Table 3). In both the AU and NZ validation cohorts, we found an advantage in the accuracy of predicting BW250 using the 600k panel over the 40k SNP panel.

Residual feed intake and BW250 were also predicted using a reference population comprising data solely from $\mathrm{AU}$ or from NZ. When the reference population was exclusively from AU, the prediction of RFI in NZ heifers was low or zero ( -0.01 to 0.03 ; Table 2$)$. When the reference population was exclusively NZ, the accuracy of prediction in AU heifers was slightly higher (0.11 to 0.21$)$ with a marked advantage of the $600 \mathrm{k}$ SNP panel over the 40k SNP panel. The Bayesian methods gave the greatest advantage in this situation. Prediction of BW250 using a single-country reference population was similar when either AU or NZ was the validation population (Table 3 ).

The degree or strength of relationship between individuals in the reference population is likely to affect the accuracy of genomic predictions. For this reason, the genomic relationship matrix $(\mathbf{G})$ was investigated further. In Figure 1, each individual square represents the proportion of the genome that a heifer shares with another heifer in the experiment. An example of this is that half sisters will have approximately 0.25 of their respective SNP alleles in common. Figure 1 shows that heifers in the AU trials were more related to each other than to the NZ heifers. Within the AU heifers, the degree of relationship was constant, and the average

Table 2. Accuracies of genomic estimated breeding values using 40,000 (40k)- and 600,000 (600k)-SNP panels for residual feed intake estimated using genomic BLUP (GBLUP), BayesA, and BayesMulti ${ }^{1}$

\begin{tabular}{|c|c|c|c|c|c|c|c|c|}
\hline Validation & n (Ref) & $\mathrm{n}(\mathrm{Val})$ & \multicolumn{2}{|c|}{ GBLUP } & \multicolumn{2}{|c|}{ BayesA } & \multicolumn{2}{|c|}{ BayesMulti } \\
\hline AU1 & 1,504 & 278 & 0.29 & 0.28 & 0.41 & 0.40 & 0.39 & 0.41 \\
\hline AU3 & 1,483 & 299 & 0.30 & 0.29 & 0.41 & 0.42 & 0.42 & 0.42 \\
\hline Average & & & 0.29 & 0.29 & 0.40 & 0.41 & 0.39 & 0.41 \\
\hline NZ1 & 1,670 & 112 & 0.62 & 0.67 & 0.62 & 0.67 & 0.73 & 0.63 \\
\hline Average & & & 0.28 & 0.31 & 0.29 & 0.31 & 0.29 & 0.31 \\
\hline $\mathrm{AU}$ & 939 & 843 & 0.11 & 0.14 & 0.14 & 0.21 & 0.11 & 0.21 \\
\hline $\mathrm{NZ}$ & 843 & 939 & -0.01 & 0.01 & 0.00 & 0.03 & 0.02 & 0.03 \\
\hline
\end{tabular}

${ }^{1}$ The validation data sets (Val) were individual cohorts excluded from the reference population (Ref) used to estimate marker effects; for example, AU1 is where Australian cohort 1 is the validation data set, NZ1 is where New Zealand cohort 1 is the validation data set, and so on. 
Table 3. Accuracies of genomic estimated breeding values (GEBV) using $\sim 40,000$ (40k)- and $\sim 600,000$ (600k)-SNP panels for 250-d BW estimated using genomic BLUP (GBLUP), BayesA, and BayesMulti ${ }^{1}$

\begin{tabular}{|c|c|c|c|c|c|c|c|c|}
\hline \multirow[b]{2}{*}{ Validation } & \multirow[b]{2}{*}{ n (Ref) } & \multirow[b]{2}{*}{ n (Val) } & \multicolumn{2}{|c|}{ GBLUP } & \multicolumn{2}{|c|}{ BayesA } & \multicolumn{2}{|c|}{ BayesMulti } \\
\hline & & & $40 \mathrm{k}$ & $600 \mathrm{k}$ & $40 \mathrm{k}$ & $600 \mathrm{k}$ & $40 \mathrm{k}$ & $600 \mathrm{k}$ \\
\hline AU3 & 1,483 & 299 & 0.36 & 0.40 & 0.38 & 0.44 & 0.39 & 0.43 \\
\hline Average & & & 0.35 & 0.38 & 0.37 & 0.41 & 0.35 & 0.40 \\
\hline NZ1 & 1,670 & 112 & 0.61 & 0.61 & 0.61 & 0.60 & 0.69 & 0.59 \\
\hline Average & & & 0.22 & 0.24 & 0.24 & 0.25 & 0.27 & 0.25 \\
\hline $\mathrm{AU}$ & 939 & 843 & 0.07 & 0.09 & 0.09 & 0.11 & 0.09 & 0.11 \\
\hline NZ & 843 & 939 & 0.09 & 0.11 & 0.09 & 0.13 & 0.11 & 0.12 \\
\hline
\end{tabular}

${ }^{1}$ The validation data sets (Val) were individual cohorts excluded from the reference population (Ref) used to estimate marker effects; for example, AU1 is where Australian cohort 1 is the validation data set, NZ1 is where New Zealand cohort 1 is the validation data set, and so on.

proportion of SNP alleles that were shared was 0.13. The average proportion of SNP alleles that were shared between $\mathrm{AU}$ and $\mathrm{NZ}$ animals was 0.07. Within the NZ group of heifers, the average relationship was similar to that of Australian heifers (0.12); however, the relationships among heifers were more variable. This could reflect larger groups of heifers sired by the same bull and could demonstrate that different strains existed in the NZ population, with more or less North American Holstein genetics. To determine if this was the case, a principal component (PC) analysis was performed on the genomic relationships. This analysis separated groups of animals that were most genetically different (Figure 2).

In Figure 2, the first (largest) and second (second largest) axis of variation, $\mathrm{PC} 1$ and $\mathrm{PC} 2$, separated $\mathrm{AU}$ and NZ heifers, confirming that these groups are genetically different. The third axis (PC3) separated the NZ heifers with an increasing proportion of North American Holstein genetics to the right of the graph. The AU heifers lie almost, but not quite, to the extreme right of this graph, indicating they had a high proportion of North American Holstein genetics but not as high as a small group of NZ heifers.

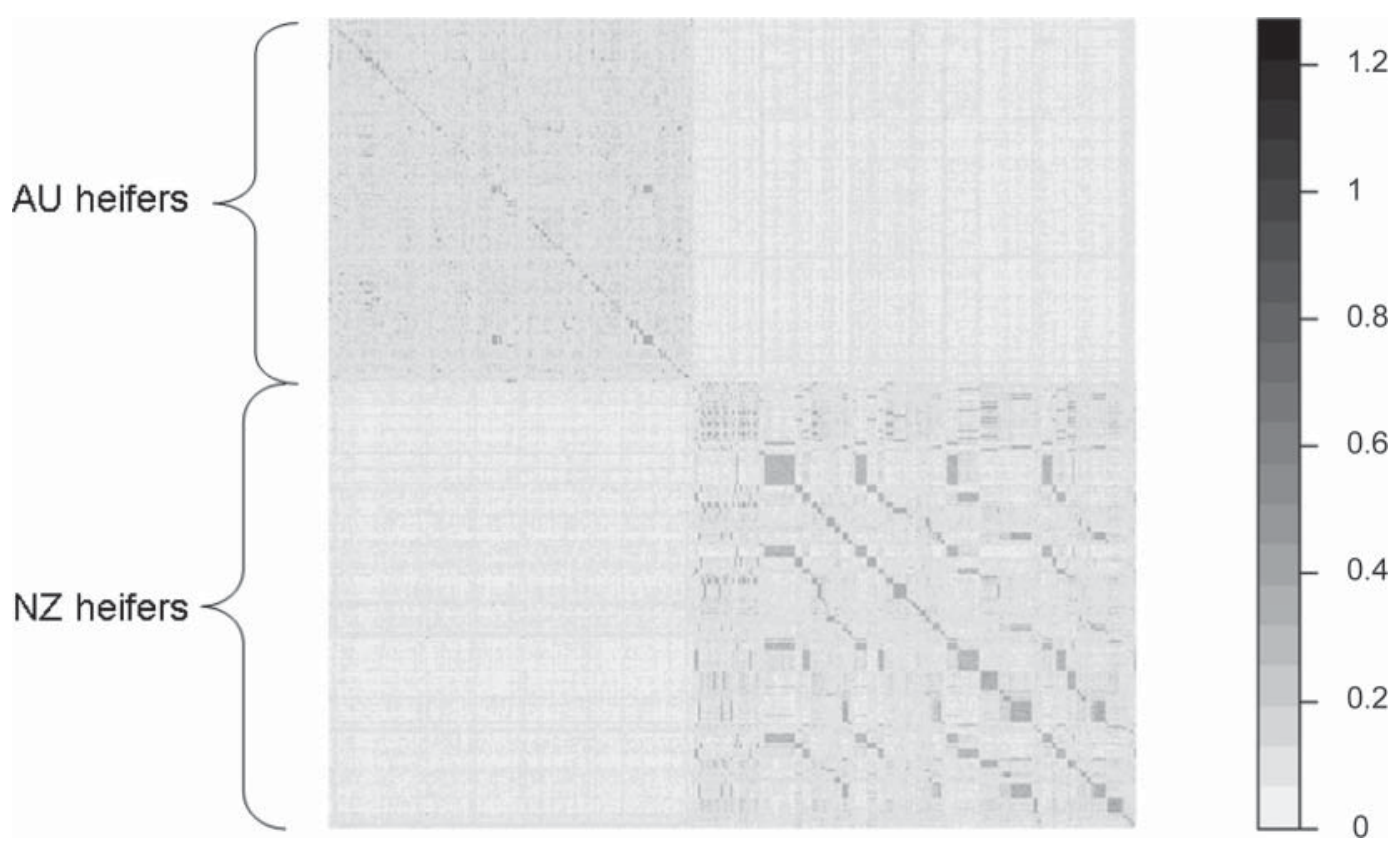

Figure 1. Genomic relationships among heifers in the trial, derived from the 625,000 SNP genotypes for each heifer. Each individual square is the proportion of the genome that a heifer shares with another heifer. Heifers are ordered across the rows and across the columns by country, then by trial, then by sire. Australian (AU) heifers are first, New Zealand (NZ) heifers follow. The intensity of black indicates the degree of relationship: the darker the square, the closer the relationship. 

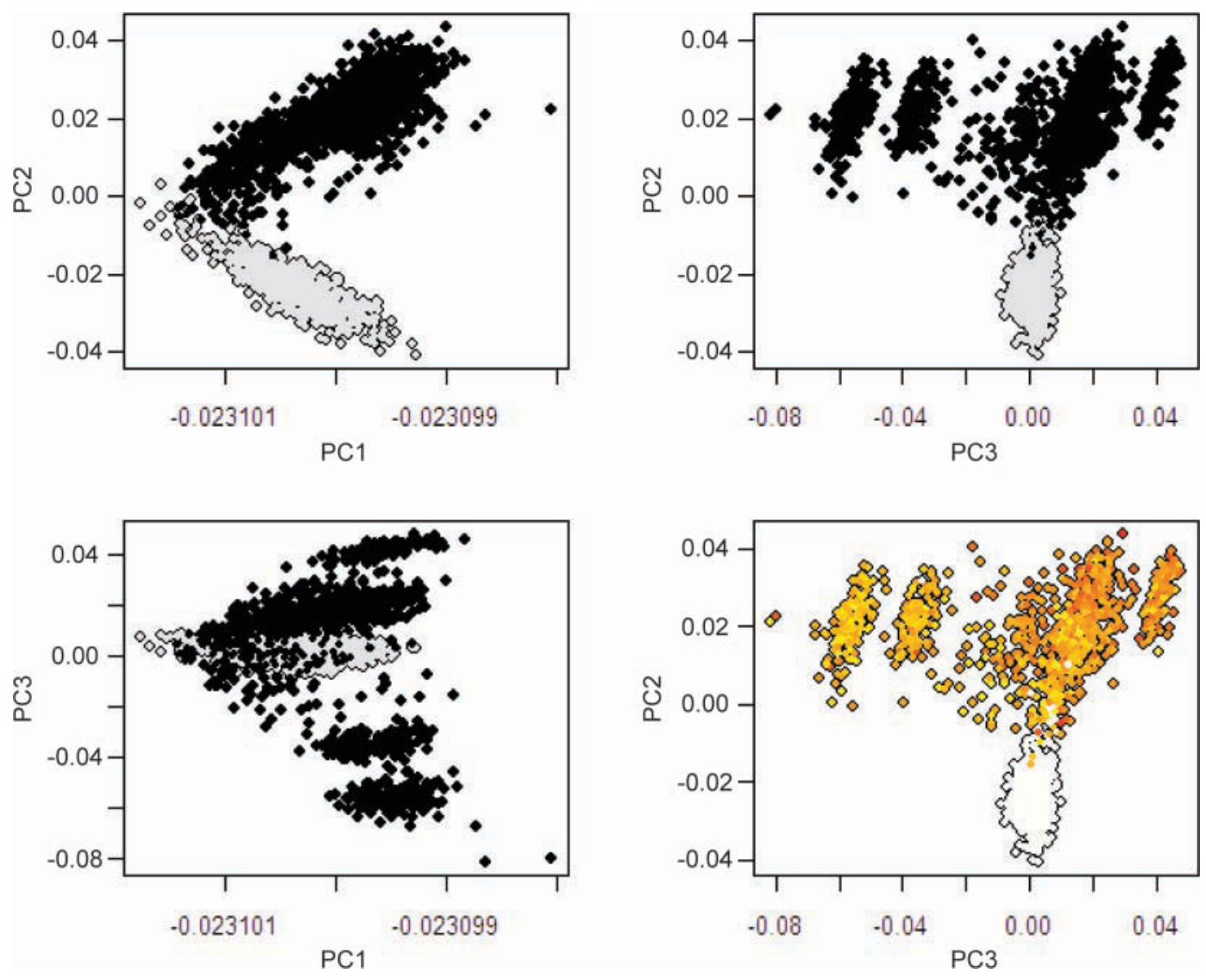

Figure 2. Principal component analysis of the genomic relationships among the heifers tested for residual feed intake (RFI). Australian heifers are represented by lighter dots and New Zealand (NZ) heifers are represented by darker dots in the first 3 panels. In the fourth (lower right hand corner), the dots representing NZ heifers are colored and the dots for Australian heifers are white. The intensity of color indicates the proportion of North American Holstein genetics; that is, red dots are animals with a high proportion of recorded North American ancestry. Color version available in the online PDF.

\section{GWAS}

Only 1 SNP for BW250 was detected to be among the largest 1,000 SNP effects in both AU and NZ data sets using either BayesA or BayesMulti and it was located at 25,015,640 bp on chromosome 14. The BayesMulti effects for chromosome 14 are shown in Figure 3. The BayesA effects were similar; in fact, the 1,000 largest SNP effects, ordered by the absolute value of the SNP effect, were the same when estimated using BayesA and BayesMulti, and the correlations (of all SNP effect estimates) between the 2 methods were 0.95 for RFI and 0.96 for BW250. As expected, when the AU and NZ BW250 data were combined, the same peak existed at around $25 \mathrm{Mb}$ (not shown). Again, the SNP located at 25,015,640 bp was among the largest 1,000 SNP effects ordered by the absolute value of the SNP effect. Thus, an allele of the SNP located at 25,015,640 bp had a negative effect on both RFI and BW250 that was consistent in the $\mathrm{NZ}$ and $\mathrm{AU}$ data sets, indicating that a putative QTL in this region may be associated with smaller body size and lower RFI (i.e., greater effi- ciency). However, another even larger QTL "peak" was located between 30 and $40 \mathrm{Mb}$ for RFI. When ranked by SNP effect size, the 8 largest effects on RFI were SNP located from $35,658,850$ to $35,723,295 \mathrm{bp}$.

\section{DISCUSSION}

Using around 625,000 SNP per heifer and a data set of 1,782 growing dairy heifers (from AU and NZ), it was possible to predict RFI with an accuracy of 0.31 (in $\mathrm{NZ}$ ) and 0.37 (in AU). Although across-country prediction was poor, the accuracy of genomic prediction of RFI (in particular) was higher when a 600k SNP panel was used instead of a 40k SNP panel. This can clearly be seen when the reference population was exclusively NZ to predict GEBV of AU heifers for RFI.

\section{Genetic Parameters}

The heritabilities of RFI estimated using a genomic relationship matrix (0.25 and 0.41 in AU and NZ data, 
a)

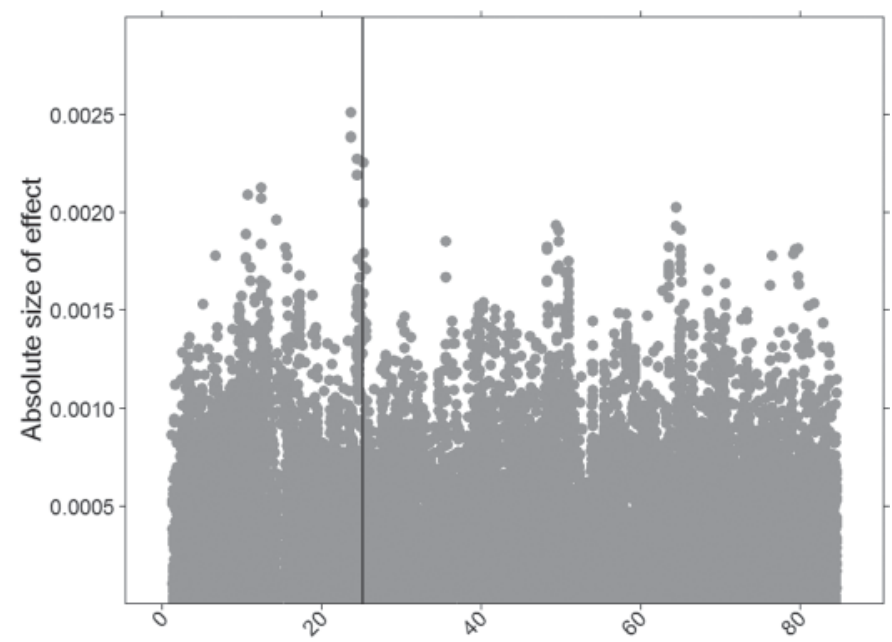

b)

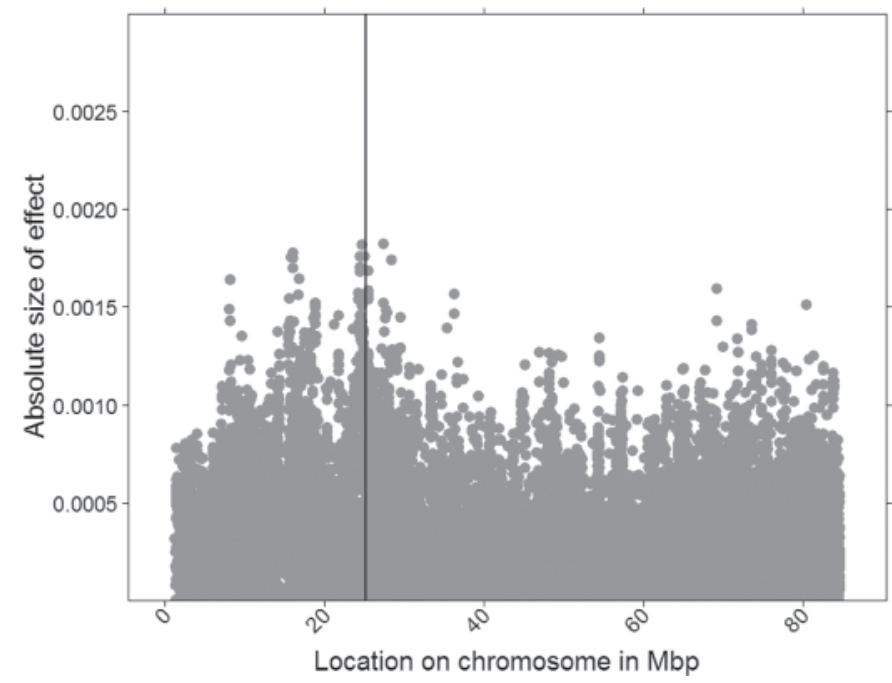

Figure 3. Single nucleotide polymorphism effects for 250-d BW $(\mathrm{kg})$ from BayesMulti on chromosome 14 estimated using heifers from either (a) Australia or (b) New Zealand. The position of the PLAG1/ CHCHD7 and RDHE2 clusters of genes is shown with a black vertical bar.

respectively; Table 1) were comparable to heritabilities estimated using pedigree relationship matrices and the same data (0.27 in AU; Williams et al., 2011). Other studies have reported similar estimates; for example, in 377 lactating dairy cows in Scotland, the heritability of RFI was estimated to be 0.32 (Veerkamp et al., 1995). In larger studies of around 1,300 growing Charolais (beef) bulls, the heritability of RFI was estimated to be 0.43 (Arthur et al., 2001). Differences in heritability estimates can be attributed largely to sampling. The higher heritability of RFI in the NZ data could be because the genetic variation in that population included variation between the 2 strains (of high percentage
North American Holstein and low percentage North American Holstein; as indicated in Figure 2).

It was not possible to precisely estimate the genetic correlation of RFI in AU and NZ, when RFI was considered a different trait in AU and NZ (using a bivariate analysis). The same was true of BW250. However, the AIC had its lowest value when the genetic correlation between countries was fixed at close to 1 . This suggests that the correlations of BW250 and RFI between AU and NZ were likely to be high and positive. A strong correlation is important because it increases the prospect of successfully predicting genomic breeding values across countries.

In the present study and elsewhere, it appears that sufficient genetic variation exists in RFI to enable this trait to be considered a candidate for future dairy selection indices, provided its associated economic value is large enough for RFI to have an effect on sire rankings when included in an index of overall economic merit. Before RFI can be considered as a candidate trait in future selection indices, genetic correlations between other traits under selection are required, especially the genetic correlation between RFI and fertility. This is especially relevant because the principal calculation of RFI and energy balance is the same, as both traits are calculated from feed intake adjusted for energy requirements (Veerkamp, 2002). The distinction between RFI and energy balance in lactating dairy cows is that RFI includes an adjustment of mobilization of body reserves. Without fully accounting for this, there is a risk that selection for low RFI could result in selection of animals with lower body condition. In the experiment here, body composition data were not available. However, it is unlikely that body composition will play a key role in variation in RFI in growing heifers because they are growing rather than mobilizing large amounts of body reserves to sustain lactation.

\section{The Accuracy of Predicting Genomic Breeding Values}

The accuracy of the genomic prediction of RFI was 0.31 and 0.37 for NZ and AU data sets, respectively, when averaged across the 3 cohorts and models using the 600k SNP panel (Table 2). Using a related trait (energy balance), Verbyla et al. (2010) calculated a similar correlation between GEBV and TBV of 0.52. The accuracy was calculated by dividing the correlation between the GEBV and phenotype adjusted for fixed effects by the square root of the heritability. This adjustment was made to account for the fact that raw phenotypes were used instead of TBV. These accuracies were compared with deterministic predictions of accuracy (Daetwyler et al., 2008; Goddard, 2009) calculated using the fol- 
lowing assumptions: an effective population size of 100 (de Roos et al., 2008), 2,000 phenotypic records, and a genome length of $30 \mathrm{M}$. The theoretical predicted accuracies; that is, $\mathrm{r}(\mathrm{GEBV}, \mathrm{TBV})$ were between 0.26 and 0.34 when the heritability estimate of the trait was between 0.25 and 0.40 . This aligns closely with our results.

We found an advantage in the accuracy of genomic predictions of BayesA and BayesMulti over GBLUP for RFI in AU heifers. This was not observed in NZ heifers or for BW250. Speculatively, the genetic architecture of RFI and the genomic relationships between animals in the $\mathrm{AU}$ population could have been responsible for this discrepancy. The Bayesian methods should provide greater accuracies when some SNP are in LD with QTL of large to moderate size. For BW250 at least, it appears that many QTL of small size underlie the variation in this trait; hence, GBLUP was not superior to the Bayesian methods. Although several SNP alleles were close to the PLAG1 gene on chromosome 14 that had among the largest effects on BW250, these SNP were not associated with large enough effect sizes to affect the accuracy of genomic prediction.

The ability to predict genomic breeding values within and between countries partly depends on the strength of genomic relationships between all pair-wise combinations of individuals; the higher the level of genomic relationship among individuals, the more accurately genomic breeding values can be predicted in that population. In this data set, predicting the accuracy using a reference population from one country and a validation population comprising heifers from the other country was poor. To understand this better, the average LD determined using the $\mathrm{r}^{2}$ statistic and the correlation of gametic phase (r) in AU and NZ were calculated using the method described by de Roos et al. (2008) and in Figure 4. The average level of $\mathrm{LD}\left(\mathrm{r}^{2}\right)$ as a function of genomic distance was remarkably similar in AU and NZ Holsteins, at least at this short interval. The correlation of gametic phase (r) between AU and NZ was also high at short intervals between SNP, but declined rapidly. Given the limited ability to predict across countries that we observed, even with the $600 \mathrm{k}$ genomic data (spacing approximately $7 \mathrm{~kb}$ between SNP), gametic phase between SNP and QTL is likely lower than that between SNP across countries.

As the data consisted of phenotypes collected in $\mathrm{AU}$ and NZ, it could be argued that a bivariate model may have been a more appropriate way to estimate GEBV; that is, treating RFI and BW250 as different traits in AU and NZ. However, GEBV that were estimated for validation populations using bivariate and univariate models were very similar - correlations between estimates were $>0.99$; that is, no differences were found in GEBV according to the model fitted.

\section{SNP Panel Size}

The accuracy of prediction of GEBV was generally (but not consistently) higher when a 600k SNP panel

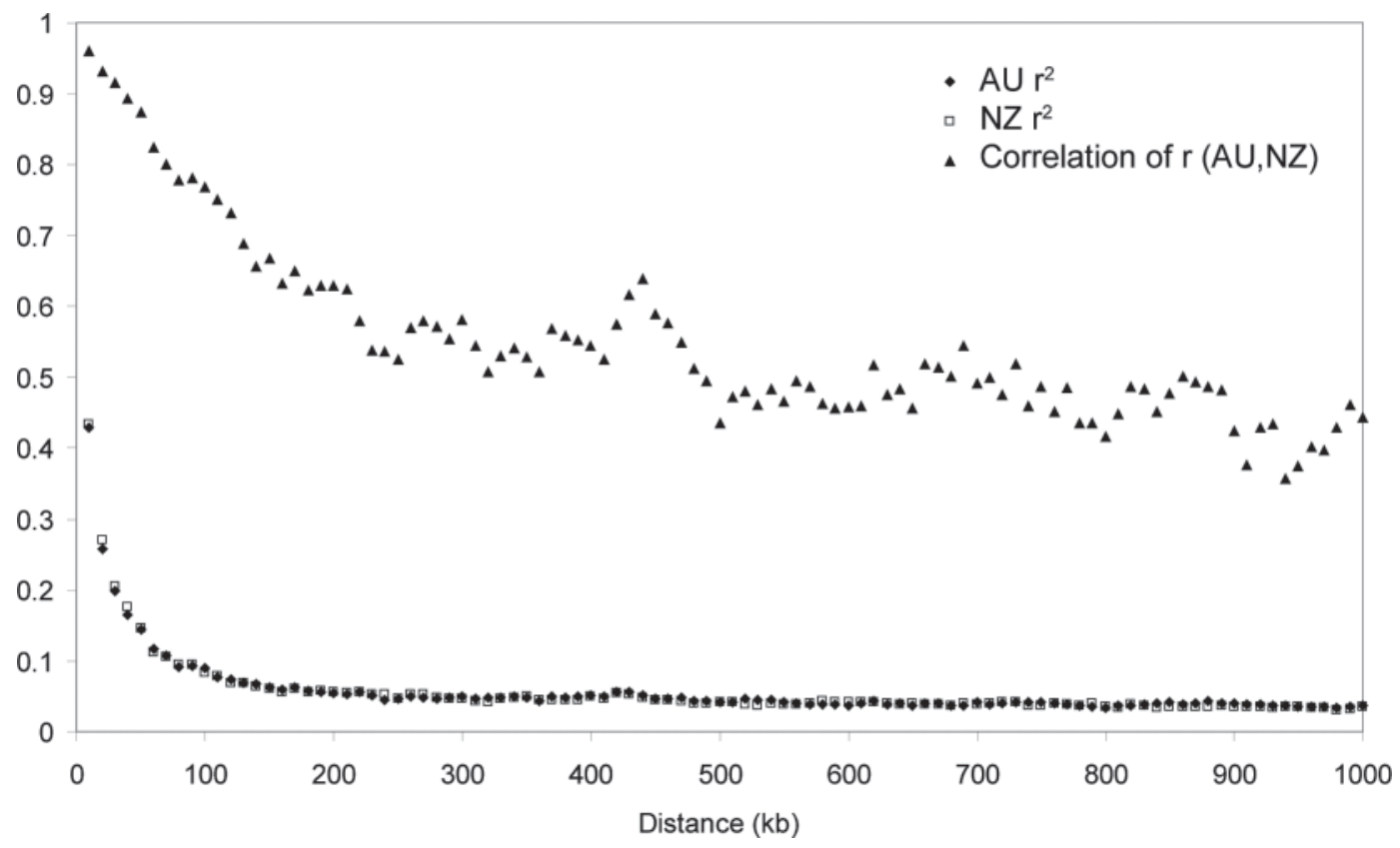

Figure 4. Average linkage disequilibrium (LD) measured as $\mathrm{r}^{2}$ as a function of genomic distance (in kb) for heifers from Australia (AU) and New Zealand (NZ) and the average relationship between gametic phase in AU and NZ heifers for marker pairs. 
was used instead of a 40k SNP panel, and the advantage of the 600k SNP panel over the 40k SNP panel was usually small. For GBLUP, BayesA, and BayesMulti, the advantage of the 600k panel over the 40k SNP panel averaged over the validation populations (AU1, AU2, AU3, NZ1, NZ2, NZ3) was 4.3, 4.7, and 3.7\%, respectively (Table 3 ). When the $40 \mathrm{k}$ SNP panel was used, the accuracy of prediction across countries ranged between -0.01 and 0.14 (Tables 2 and 3). When averaged across countries, the increase in accuracy of the $600 \mathrm{k}$ panel compared with the $40 \mathrm{k}$ panel was between 0.03 (GBLUP) and 0.06 (BayesMulti) for RFI (Table 2) and between 0.02 (BayesMulti) and 0.03 (BayesA) for BW250 (Table 3). The exception was the prediction of AU RFI GEBV using an NZ-only reference population, where the advantage of the $600 \mathrm{k}$ SNP panel was a 27 to $90 \%$ improvement in accuracy (Table 3 ).

The increased accuracy from the $600 \mathrm{k}$ panel could be because the persistence of the LD phase is much shorter across strains of Holsteins (such as AU and NZ) than within strains. Therefore, a 600k SNP panel could be better at capturing the LD between a SNP and a QTL across strains. The reason why a reference population to predict NZ GEBV did not show the same advantage could be because the same population admixture or diversity seen in the NZ population (Figure 2) did not exist in the AU Holstein population. Therefore, in this instance, no extra QTL were captured by using a $600 \mathrm{k}$ instead of a 40k SNP panel. The genetic composition of $\mathrm{AU}$ and $\mathrm{NZ}$ dairy calves was quite different and could therefore require denser markers to capture QTL-SNP associations that persist across the 2 countries. For example, the genomic relationships estimated using SNP data (Figure 2; boxes 2-4) demonstrated (1) that the AU heifers were genetically homogeneous compared with the NZ heifers, and (2) that a group of NZ heifers existed that was genetically similar to the AU heifers (those that had a high proportion of recent North American Holstein ancestry), but another group existed that was genetically dissimilar (Figure 2, box 4). This could reflect the fact that many modern New Zealand Holsteins are graded up from a Jersey cow base or even the closed Holstein population founded on animals imported from the United States in the 1920s and closed until the 1960s (Harris and Kolver, 2001). Modern NZ "strains" of Holsteins typically have lower BW, lower levels of milk production, and better levels of fertility than their North American counterparts (Harris and Kolver, 2001; Macdonald et al., 2008). The results reported here also confirm the observation of de Roos et al. (2008) that genomic relationships between NZ Holsteins and other Holstein populations were low. The fact that more variation was observed in the NZ heifers, as identified using the principal component analysis and shown in Figure 2, could partly explain the slightly lower accuracies of GEBV for BW250 and RFI in the NZ data.

\section{Detection of QTL}

Given the underlying population structure; namely, the predominance of North American Holstein genetics in $\mathrm{AU}$ heifers and mixed origin for NZ heifers, it was interesting to observe similar QTL in both populations. Although several regions were found where putative QTL were detected, a SNP was found that appeared

a)

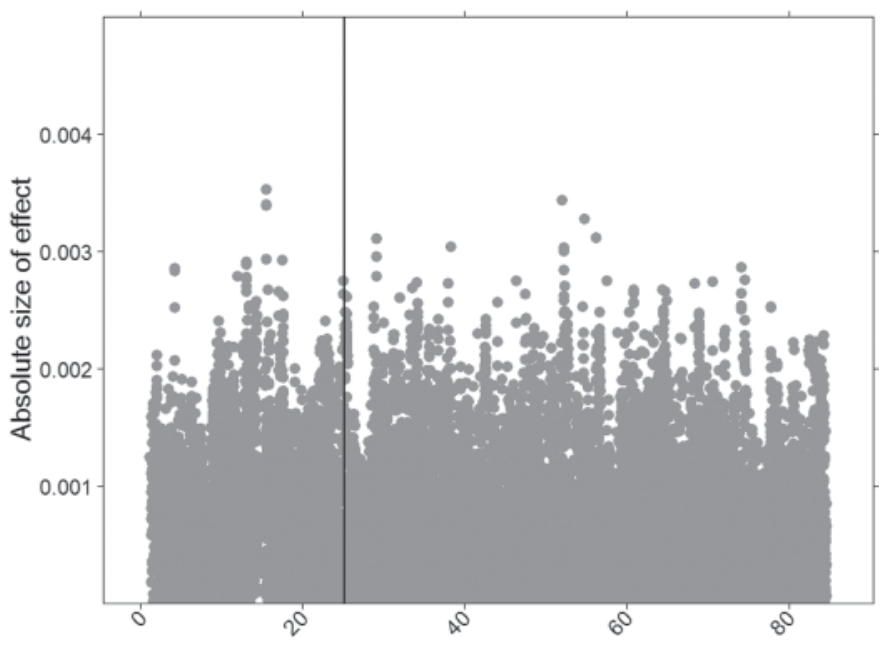

b)

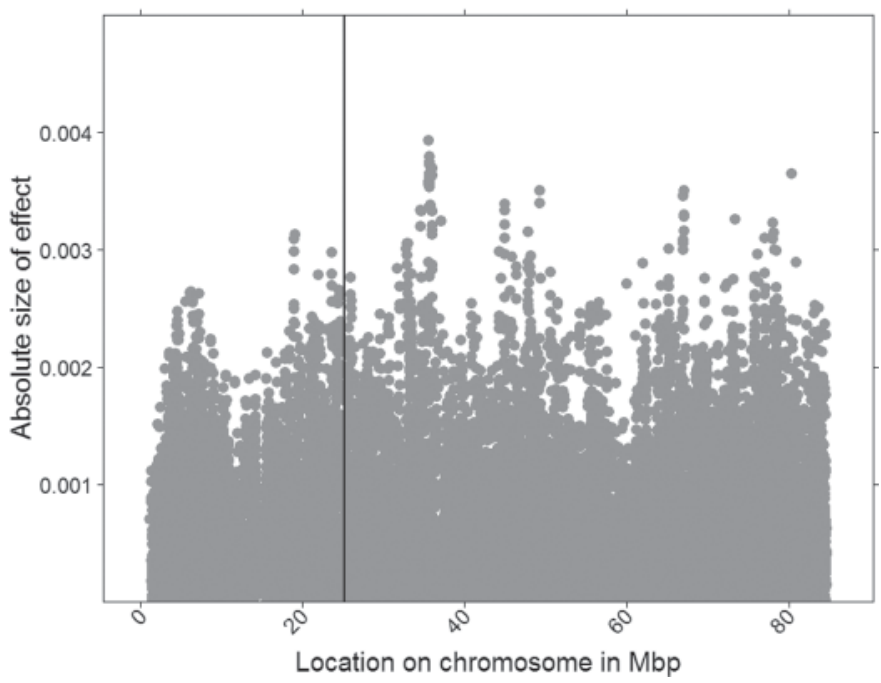

Figure 5. Single nucleotide polymorphism effects for residual feed intake (RFI) from BayesMulti on chromosome 14 estimated using heifers from either (a) Australia or (b) New Zealand. The position of the PLAG1/CHCHD7 and RDHE2 clusters of genes is shown with a black vertical bar. 
to be in LD with a QTL that had an effect on BW250 among the largest 1,000 SNP effects (ordered by the absolute value of the SNP effect) in both NZ and AU heifers (Figure 3). This SNP was positioned at 25,015,640 bp on chromosome 14. The "peak" surrounding this SNP is shown in Figure 3 and coincides with the position of a cluster of genes (PLAG1, CHCHD \%, and RDHE2) believed to be associated with human stature (Gudbjartsson et al., 2008; Kim et al., 2010) and close to SNP that have a large effect on stature in beef and dairy cattle (Karim et al., 2011; Pryce et al., 2011). The position of PLAG1 is $25,005,800$ to $25,010,800 \mathrm{bp}$, CHCHD7 is $25,048,500$ to $25,063,200 \mathrm{bp}$, and RDHE2 is $25,134 \mathrm{k}$ to $25,199 \mathrm{k} \mathrm{bp}$. We also observed a peak in a very similar position for RFI (Figure $5 \mathrm{~b}$ ). In fact, SNP in this region have been found to have an effect on RFI in AU beef cattle (S. Bolormaa, Department of Primary Industries, Melbourne, Australia; personal communication, 2011). However, a study of a subset of this data set comprising beef animals failed to find significant associations in this region, although a SNP at 17 to $18 \mathrm{Mb}$ was significant $(P=0.0005)$ (Bolormaa et al., 2011). Nkrumah et al. (2007b) found a significant relationship between RFI in beef cattle and a SNP located at another location $(74 \mathrm{Mb})$ on the same chromosome.

Another larger QTL associated with RFI was located on chromosome 14 at around $36 \mathrm{Mb}$. The closest gene to the location of these SNP is NCOA2 (also known as TIF2), which has a role in controlling energy balance between white and brown adipose tissues in mice (Picard et al., 2002). Mice that are homozygous recessive (Tif2 ${ }^{-/}$) are protected against obesity and appear to have enhanced adaptive thermogenesis (Picard et al., 2002). In fact, Tif2 ${ }^{-/-}$mice did not become obese when they were fed a high fat diet, whereas $\mathrm{Tif2} 2^{+/+}$mice became obese. Therefore, $N C O A 2$ could play a role in a pathway controlling feed efficiency that is conserved across mammalian species.

\section{CONCLUSIONS}

These results indicate that the Illumina High Density SNP chip, in conjunction with Bayesian models that estimate SNP effects for all SNP fitted simultaneously, could be a powerful tool to detect QTL. In addition, having 2 independent data sets (from NZ and $\mathrm{AU}$ ) is an advantage because SNP that are associated with a trait in more than one population can be found.

\section{ACKNOWLEDGMENTS}

The Department of Primary Industries authors thank DPI colleagues Taffy Phillips, Marg Jenkin, Mel Porker, and Nick Linden for their technical expertise in the day- to-day running of the Rutherglen feed intake facility, the management of the calves, and data collation. The skilled assistance of other farm staff is also acknowledged. Funding was provided by the Geoffrey Gardiner Foundation (Melbourne, Australia), the Dairy Futures Cooperative Research Centre (Melbourne, Australia), and Department of Primary Industries (Victoria, Australia). The DairyNZ and LIC authors thank the Foundation for Research, Science and Technology (FRST; Wellington, New Zealand), DairyNZ (Hamilton, New Zealand), Livestock Improvement Corporation (LIC; Hamilton, New Zealand), and Trade and Enterprise (NZ) (Auckland, New Zealand) for funding, the LIC calf procurement team led by Katie Eketone and Jack Hooper, and the DairyNZ technical team at Hawera for managing the trial.

\section{REFERENCES}

Arthur, P. F., J. A. Archer, and R. M. Herd. 2004. Feed intake and efficiency in beef cattle: Overview of recent Australian research and challenges for the future. Aust. J. Exp. Agric. 44:361-369.

Arthur, P. F., J. A. Archer, D. J. Johnston, R. M. Herd, E. C. Richardson, and P. F. Parnell. 2001. Genetic and phenotypic variance and covariance components for feed intake, feed efficiency, and other postweaning traits in Angus cattle. J. Anim. Sci. 79:2805-2811.

Bolormaa, S., B. J. Hayes, K. Savin, R. Hawken, W. Barendse, P. F. Arthur, R. M. Herd, and M. E. Goddard. 2011. Genome wide association study for feedlot and growth traits in cattle. J. Anim. Sci. 89:1684-1697. http://dx.doi.org/10.2527/jas.2010-3079.

Browning, B. L., and S. R. Browning. 2009. A unified approach to genotype imputation and haplotype phase inference for large data sets of trios and unrelated individuals. Am. J. Hum. Genet. $84: 210-223$

Carnie, K. R., S. R. Davis, K. A. Macdonald, G. C. Waghorn, V. V. Obolonkin, and R. J. Spelman. 2010. Brief communication: Variation in feed conversion efficiency in Holstein-Friesian heifer calves. Proc. N.Z. Soc. Anim. Prod. 70:250-252.

Daetwyler, H. D., B. Villanueva, and J. A. Woolliams. 2008. Accuracy of predicting genetic risk of disease using a genome-wide approach. PLoS ONE 3:e3395.

de Roos, A. P., B. J. Hayes, R. J. Spelman, and M. E. Goddard. 2008. Linkage disequilibrium and persistence of phase in Holstein-Friesian, Jersey and Angus cattle. Genetics 179:1503-1512.

Gilmour, A. R., B. J. Gogel, B. R. Cullis, and R. Thompson. 2006. ASReml User Guide Release 2.0. VSN International Ltd., Hemel Hempstead, UK.

Goddard, M. E. 2009. Genomic selection: Prediction of accuracy and maximisation of long term response. Genetica 136:245-257.

Gudbjartsson, D. F., G. B. Walters, G. Thorleifsson, H. Stefansson, B. V. Halldorsson, P. Zusmanovich, P. Sulem, S. Thorlacius, A. Gylfason, S. Steinberg, A. Helgadottir, A. Ingason, V. Steinthorsdottir, E. J. Olafsdottir, G. H. Olafsdottir, T. Jonsson, K. BorchJohnsen, T. Hansen, G. Andersen, T. Jorgensen, O. Pedersen, K. K. Aben, J. A. Witjes, D. W. Swinkels, M. den Heijer, B. Franke, A. L. M. Verbeek, D. M. Becker, L. R. Yanek, L. C. Becker, L. Tryggvadottir, T. Rafnar, J. Gulcher, L. A. Kiemeney, A. Kong, U. Thorsteinsdottir, and K. Stefansson. 2008. Many sequence variants affecting diversity of adult human height. Nat. Genet. 40:609-615.

Harris, B. L., and E. S. Kolver. 2001. Review of holsteinization on intensive pastoral dairy farming in New Zealand. J. Dairy Sci. 84(E. Suppl.):E56-E61.

Hegarty, R. S., J. P. Goopy, R. M. Herd, and B. McCorkell. 2007. Cattle selected for lower residual feed intake have reduced daily methane production. J. Anim. Sci. 85:1479-1486. 
Ho, C., R. Nessler, P. Doyle, and B. Malcolm. 2005. Future dairy farming systems in irrigation regions. Aust. Farm Bus. Manage. J. 2:65-74.

Karim, L., H. Takeda, L. Lin, T. Druet, J. A. C. Arias, D. Baurain, N. Cambisano, S. R. Davis, F. Farnir, B. Grisart, B. L. Harris, M. D. Keehan, M. D. Littlejohn, R. J. Spelman, M. Georges, and W. Coppiters. 2011. Variants modulating the expression of a chromosome domain encompassing PLAG1 influence bovine stature. Nat. Genet. 43:405-413.

Kim, J.-J., H.-I. Lee, T. Park. Kim, J.-E. Lee, N. H. Cho, C. Shin, Y. S. Cho, J. Y. Lee, B. G. Han, H. W. Yoo, and J. K. Lee. 2010. Identification of 15 loci influencing height in a Korean population. J. Hum. Genet. 55:27-31.

Macdonald, K. A., G. A. Verkerk, B. S. Thorrold, J. E. Pryce, J. W. Penno, L. R. McNaughton, L. J. Burton, J. A. S. Lancaster, J. H. Williamson, and C. W. Holmes. 2008. A comparison of three strains of Holstein-Friesian grazed on pasture and managed under different feed allowances. J. Dairy Sci. 91:1693-1707.

Matukumalli, L. K., C. T. Lawley, R. D. Schnabel, J. F. Taylor, M. F. Allan, M. P. Heaton, J. O'Connell, S. S. Moore, T. P. L. Smith T. S. Sonstegard, and C. P. Van Tassell. 2009. Development and characterization of a high density SNP genotyping assay for cattle. PLoS ONE 4:e5350.

Meuwissen, T. H. E., B. J. Hayes, and M. E. Goddard. 2001. Prediction of total genetic value using genome-wide dense marker maps. Genetics 157:1819-1829.

Nieuwhof, G. J., J. A. M. van Arendonk, H. Vos, and S. Korver. 1992 Genetic relationships between feed intake, efficiency and production traits in growing bulls, growing heifers and lactating heifers. Livest. Prod. Sci. 32:189-202

Nkrumah, J. D., J. A. Basarab, Z. Wang, C. Li, M. A. Price, E. K Okine, D. H. Crews Jr., and S. S. Moore. 2007a. Genetic and phenotypic relationships of feed intake and measures of efficiency with growth and carcass merit of beef cattle. J. Anim. Sci. 85:2711-2720.

Nkrumah, J. D., E. L. Sherman, C. Li, E. Marques, D. H. Crews Jr., R. Bartusiak, B. Murdoch, Z. Wang, J. A. Basarab, and S. S. Moore. 2007b. Primary genome scan to identify putative quantitative trait loci for feedlot growth rate, feed intake, and feed efficiency of beef cattle. J. Anim. Sci. 85:3170-3181.

Picard, F., M. Gehin, J.-S. Annicotte, S. Rocchi, M.-F. Champy, B. W. O'Malley, P. Chambon, and J. Auwerx. 2002. SRC-1 and TIF2 control energy balance between white and brown adipose tissues. Cell 111:931-941.

Pryce, J. E., S. Bolormaa, A. J. Chamberlain, P. J. Bowman, K. Savin, M. E. Goddard, and B. J. Hayes. 2010. A validated genome wide association study in two dairy cattle breeds for variable length haplotypes. J. Dairy Sci. 93:3331-3345.

Pryce, J. E., B. J. Hayes, S. Bolormaa, and M. E. Goddard. 2011. Polymorphic regions affecting human height also control stature in cattle. Genetics 187:981-984.

Simm, G., R. F. Veerkamp, and P. Persaud. 1994. The economic performance of dairy cows of different predicted genetic merit for milk solids production. Anim. Prod. 58:313-320.

VanRaden, P. M. 2008. Efficient methods to compute genomic predictions. J. Dairy Sci. 91:4414-4423.

Veerkamp, R. F. 2002. Feed intake and energy balance in lactating animals. Session 10-01 in Proc. 7th World Congr. Gen. Appl. Livest. Prod., Montpellier, France. CD-ROM. 7th WCGALP INRA and CIRAD, Montpellier, France.

Veerkamp, R. F., G. C. Emmans, A. R. Cromie, and G. Simm. 1995. Variance components for residual feed intake in dairy cows. Livest. Prod. Sci. 41:111-120.

Verbyla, K. L., M. P. L. Calus, H. A. Mulder, Y. de Haas, and R. F. Veerkamp. 2010. Predicting energy-balance for dairy cows using high-density single nucleotide polymorphism information. J. Dairy Sci. 93:2757-2764.

Verbyla, K. L., B. J. Hayes, P. J. Bowman, and M. E. Goddard. 2009. Accuracy of genomic selection using stochastic search variable selection in Australian Holstein Friesian dairy cattle. Genet. Res. (Camb.) 91:307-311.

Waghorn, G. C., K. A. Macdonald, Y. Williams, S. R. Davis, and R. J. Spelman. 2012. Measuring residual feed intake in dairy heifers fed an alfalfa (Medicago sativa) cube diet. J. Dairy Sci. 95:1462-1471.

Williams, Y. J., J. E. Pryce, C. Grainger, W. J. Wales, N. Linden, M. Porker, and B. J. Hayes. 2011. Variation in residual feed intake in Holstein Friesian dairy heifers in southern Australia. J. Dairy Sci. 94:4715-4725.

Yang, J., B. Benyamin, B. P. McEvoy, S. Gordon, A. K. Henders, D. R. Nyholt, P. A. Madden, A. C. Heath, N. G. Martin, G. W. Montgomery, M. E. Goddard, and P. M. Visscher. 2010. Common SNP explain a large proportion of the heritability for human height Nat. Genet. 42:565-569. 